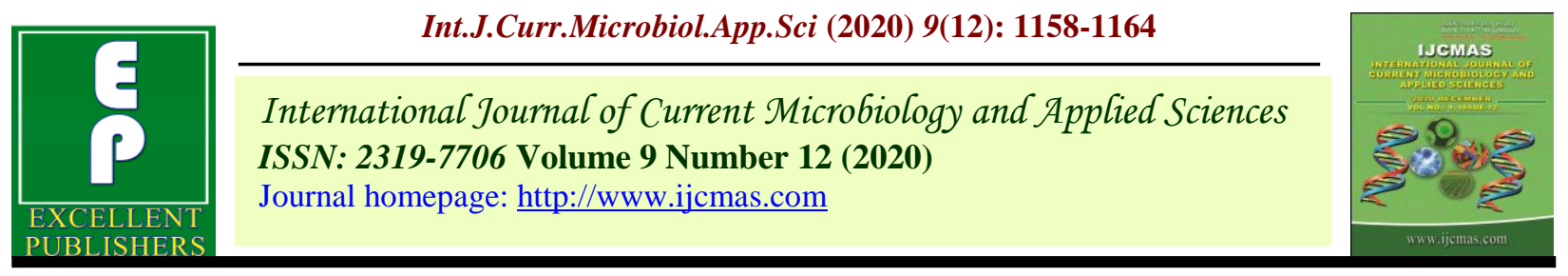

Original Research Article

https://doi.org/10.20546/ijcmas.2020.912.140

\title{
Studies on Correlation and Path Analysis for Grain Yield and its Components in Maintainer Lines of Pearl Millet [Pennisetum glaucum (L.) R. Br.]
}

\author{
Sundar Lal Dadarwal", Shyam Singh Rajput and Giradhari Lal Yadav
}

Department of Plant Breeding and Genetics, Sri Karan Narendra Agriculture University Jobner, Jaipur, Rajasthan-303329, India

*Corresponding author

\section{A B S T R A C T}

\section{Keywords}

Genotypic

correlation, Path coefficient, Pearl millet, Phenotypic correlation

Article Info

Accepted:

10 November 2020

Available Online:

10 December 2020
A field experiment of thirty maintainer lines of pearl millet had done with an aim to study correlation and path analysis for grain yield and its yield contributing characters so as to identify maintainer lines with desirable attributes for the utilization in crop improvement program during kharif 2019. Correlation studies revealed that grain yield per plant had significant and positive association with ear head length, ear head girth, 1000-grain weight, biological yield per plant at genotypic as well as phenotypic level and harvest index at phenotypic level only. Path coefficient analysis revealed that the ear head length, biological yield per plant and harvest index were the most important characters for selection of high yielding genotypes as they exerted highest positive direct effect as well as positive correlation with grain yield per plant.

\section{Introduction}

Pearl millet popularly known as bajra is an important coarse grain cereal, a member of the Poaceae family and genus Pennisetum. It is chromosome number $2 n=14$. It is believed to have originated in West Africa (Vavilov, 1950). It is highly cross-pollinated crop with protogynous flowering and wind-borne pollination mechanism, which fulfils the essential biological requirement for hybrid development. Pearl millet is an important cultivated cereal in the world, having sixth rank after rice, wheat, maize, barley and sorghum in terms of area. It is grown on about 30 million hectares in more than thirty countries. The majority of this area is in Asia, Africa and Americas (Gupta et al., 2015). In India, pearl millet is the fourth most widely cultivated food crop after rice, wheat and maize. It occupies 7.4 million hectares with an average production of 9.13 million tonnes and the productivity of $1237 \mathrm{~kg} / \mathrm{ha}$ (Anonymous, 2019). It is consumed as both 
feed and fodder for livestock. Pearl millet is very rich in calories, proteins $(6-15 \%)$, fat (5$6 \%)$, carbohydrates $(60-72 \%)$, fibre $(1-1.8 \%)$ and minerals with less amount of $\mathrm{HCN}$, which makes it highly nutritive and palatable crop in comparison with other crops (Fleck, 1981). Correlation and path co-efficient analysis are the important biometrical technique to determine the yield components. The characters that are positively correlated with yield are considerably important to plant breeder for selection purpose. Correlation provides a measure of genetic association between the characters and reveals the traits that might be useful as an index of selection. Although the correlation coefficient indicates the nature of association among the different traits, path analysis splits the correlation coefficient into measure of direct and indirect effects thus providing understanding of the direct and indirect contribution of each character towards yield. Hence, the present study was under taken to unravel the correlation and path co-efficient of grain yield and yield attributing traits in maintainer lines of pearl millet.

\section{Material and Methods}

The present investigation was carried out at Research Farm of Sri Karan Narendra Agriculture University Jobner, Jaipur, Rajasthan during kharif 2019. The place of experimentation was situated at 435 meters above mean sea level on latitude $26^{0} 05^{\prime} \mathrm{N}$ and longitude $75^{\circ} 28^{\prime} \mathrm{E}$ under agro climatic zone III A (semi- arid eastern plain zone). The experiment was laid down in randomized block design (RBD) with three replications. Each genotype was planted in a plot of $4.0 \mathrm{~m}$ $\mathrm{x} 1.0 \mathrm{~m}$ size accommodating two rows spaced $50 \mathrm{~cm}$ apart. The plant to plant spacing of 15 $\mathrm{cm}$ was maintained. The observations were recorded on five randomly selected competitive plants from each maintainer line in each replication for all the characters except days to $50 \%$ flowering and days to maturity which were recorded on plot basis. The genotypic and phenotypic correlation coefficients were calculated from the genotypic and phenotypic components of variance and covariance as described by Singh and Choudhary (1985) and as per formula given by Johnson et al., (1955). Path analysis was done as per the procedure outlined by Dewey and Lu (1959).

\section{Results and Discussion}

The awareness of correlation coefficients in all possible combinations between the yield characters were estimated at genotypic and phenotypic levels are presented in Table 1. The genotypic correlation coefficient was higher than the respective phenotypic correlation coefficient. Lower phenotypic correlations may result from the modifying effect of environment on the association of characters at the genetic level. Genotypic correlation coefficient indicates a measure of genetic association between characters and therefore, help in identifying the character and need to be considered for improvement of grain yield. As there is no suitable test of significance available for genetic correlation, their main usefulness is in strengthening the interpretations based on correlations (Pandey and Gritton, 1975). In present investigation, grain yield per plant was positively associated with all the attributes studied viz., days to $50 \%$ flowering, days to maturity, plant height, effective tillers per plant, ear head length, ear head girth, 1000-grain weight, biological yield per plant and harvest index at genotypic as well as phenotypic level. However, positively significant association of grain yield per plant was observed with ear head length $\left(r_{g}=0.962, r_{p}=0.738\right)$, ear head girth $\left(r_{g}=0.858, \quad r_{p}=0.618\right), 1000$-grain weight $\left(r_{g}=0.998, r_{p}=0.864\right)$ and biological yield per plant $\left(r_{g}=0.908, r_{p}=0.806\right)$ at genotypic as well as phenotypic levels; and, harvest index $\left(r_{\mathrm{p}}=0.420\right)$ at phenotypic level only. A significant association between two or more 
characters may implicit that the biosynthetic leading to development of these traits share some common precursor; or may be produced due to pleiotropic gene action; or may arise from linkage of the genes governing these characters. Similar results were recorded by Arulselvi et al., (2008), Govindaraj et al., (2009), Abuali et al., (2012), Patil et al., (2018) and Rasitha et al., (2019).

Days to $50 \%$ flowering had positive and significant association with days to maturity $\left(r_{\mathrm{g}}=0.993, \mathrm{r}_{\mathrm{p}}=0.932\right)$ at genotypic as well as phenotypic level.

Days to maturity recorded positive and significant association with plant height $\left(r_{g}=0.367\right)$ at genotypic level only.

Plant height exhibited positive and significant association with effective tillers per plant $\left(r_{g}\right.$ $=0.571$ ) at phenotypic level only, whereas, ear head length $\left(r_{g}=0.795, r_{p}=0.646\right)$, ear head girth $\left(r_{g}=0.705, r_{p}=0.397\right), 1000$ grain weight $\left(r_{g}=0.816, r_{p}=0.693\right)$, biological yield per plant $\left(r_{g}=0.999, r_{p}=0.858\right)$ at both genotypic and phenotypic levels.

Effective tillers per plant recorded a positive and significant association with ear head length $\left(r_{g}=0.418\right)$ and biological yield per plant $\left(r_{\mathrm{g}}=0.471\right)$ at genotypic level only, while negatively significant with harvest index $\left(r_{g}=-0.419\right)$ at genotypic level.

Ear head length had a positive and significant correlation with plant height $\quad\left(r_{g}=0.795\right.$, $\left.r_{p}=0.646\right)$, ear head girth $\left(r_{g}=0.971, r_{p}=0.640\right)$, 1000 grain weight $\left(r_{g}=0.931, r_{p}=0.790\right)$, biological yield per plant $\left(r_{g}=0.819, r_{p}\right.$ $=0.590)$ and grain yield per plant $\left(r_{g}=0.962\right.$, $r_{p}=0.738$ ) at both genotypic and phenotypic levels and effective tillers per plant $\left(r_{g}\right.$ $=0.418)$ and harvest index $\left(r_{\mathrm{g}}=0.496\right)$ at genotypic level only. Ear head girth exhibited a positive and significant correlation with plant height $\left(r_{g}=0.705, r_{p}=0.397\right)$, ear head length $\quad\left(r_{g}=0.971, \quad r_{p}=0.640\right), 1000$ grain weight $\left(r_{\mathrm{g}}=0.931, \mathrm{r}_{\mathrm{p}}=0.699\right)$, biological yield per plant $\left(r_{g}=0.630, r_{p}=0.465\right)$ and grain yield per plant $\left(\mathrm{r}_{\mathrm{g}}=0.858, \quad \mathrm{r}_{\mathrm{p}}=0.618\right)$ at both genotypic and phenotypic levels; and, harvest index $\left(r_{g}=0.711\right)$ at genotypic level only.

1000 grain weight recorded positive and significant association with plant height $\left(r_{g}=0.816, \quad r_{p}=0.693\right)$, ear head length $\left(r_{g}=0.931, \quad r_{p}=0.790\right)$, ear head girth $\left(r_{\mathrm{g}}=0.931, \mathrm{r}_{\mathrm{p}}=0.699\right)$, biological yield per plant $\left(r_{g}=0.814, r_{p}=0.728\right)$ and grain yield per plant $\left(r_{g}=0.998, r_{p}=0.864\right)$ at both genotypic and phenotypic levels; and, harvest index $\left(r_{g}=579\right)$ at genotypic level only.

Biological yield per plant showed positive and significant association with plant height $\left(r_{g}=0.999, \quad r_{p}=0.858\right)$, ear head length $\left(r_{g}=0.819, \quad r_{p}=0.590\right)$, ear head girth $\left(r_{g}=0.630, r_{p}=0.465\right), 1000$ grain weight $\left(\mathrm{r}_{\mathrm{g}}=0.814, \mathrm{r}_{\mathrm{p}}=0.728\right)$ and grain yield per plant $\left(r_{g}=0.908, r_{p}=0.806\right)$ at both genotypic as well as phenotypic levels; and effective tillers per plant $\left(r_{\mathrm{g}}=0.471\right)$ at genotypic level only.

Harvest index exhibited positive and significant association with grain yield per plant $\left(\mathrm{r}_{\mathrm{p}}=0.420\right)$ at phenotypic level; and, ear head length $\left(r_{g}=0.496\right)$, ear head girth $\left(r_{g}=0.711\right)$ and 1000 -grain weight $\left(r_{g}=0.579\right)$ at genotypic level. It exhibited negative and significant association with effective tillers per plant $\left(r_{\mathrm{g}}=-0.419\right)$ at genotypic level.

Thus, the present investigation exposed that yield component characters such as ear head length, ear head girth, 1000 grain weight and biological yield per plant exhibited significantly positive association with grain yield per plant at genotypic as well as phenotypic levels; and, harvest index at phenotypic level only. Therefore, selection based on these traits may prove rewarding towards yield improvement in pearl millet. 
Table.1 Genotypic and phenotypic correlation coefficients between different characters in maintainer lines of pearl millet

\begin{tabular}{|c|c|c|c|c|c|c|c|c|c|c|c|}
\hline Characters & & $\begin{array}{c}\text { Days to } \\
50 \% \\
\text { flowering }\end{array}$ & $\begin{array}{l}\text { Days to } \\
\text { maturity }\end{array}$ & $\begin{array}{l}\text { Plant } \\
\text { height } \\
\text { (cm) }\end{array}$ & $\begin{array}{c}\text { Effective } \\
\text { tillers/plant }\end{array}$ & $\begin{array}{c}\text { Ear head } \\
\text { length } \\
(\mathrm{cm})\end{array}$ & $\begin{array}{l}\text { Ear head } \\
\text { girth } \\
(\mathrm{cm})\end{array}$ & $\begin{array}{c}1000 \\
\text { grain } \\
\text { weight } \\
\text { (g) }\end{array}$ & $\begin{array}{l}\text { Biologica } \\
\text { l yield/ } \\
\text { plant (g) }\end{array}$ & $\begin{array}{c}\text { Harvest } \\
\text { Index } \\
(\%)\end{array}$ & $\begin{array}{c}\text { Grain } \\
\text { yield/ } \\
\text { plant (g) }\end{array}$ \\
\hline $\begin{array}{l}\text { Days to } 50 \% \\
\text { flowering }\end{array}$ & $\begin{array}{l}r_{g} \\
r_{p}\end{array}$ & $\begin{array}{l}1 \\
1\end{array}$ & $\begin{array}{l}0.993 * * \\
0.932 * *\end{array}$ & $\begin{array}{l}0.303 \\
0.263\end{array}$ & $\begin{array}{l}0.118 \\
0.093\end{array}$ & $\begin{array}{l}0.214 \\
0.075\end{array}$ & $\begin{array}{l}0.087 \\
0.052\end{array}$ & $\begin{array}{l}0.173 \\
0.190\end{array}$ & $\begin{array}{l}0.169 \\
0.151\end{array}$ & $\begin{array}{l}0.175 \\
0.050\end{array}$ & $\begin{array}{l}0.223 \\
0.168\end{array}$ \\
\hline Days to maturity & $\begin{array}{l}r_{g} \\
r_{p}\end{array}$ & & $\begin{array}{l}1 \\
1\end{array}$ & $\begin{array}{c}0.367 * \\
0.286\end{array}$ & $\begin{array}{l}0.153 \\
0.061\end{array}$ & $\begin{array}{l}0.258 \\
0.074\end{array}$ & $\begin{array}{l}0.051 \\
0.023\end{array}$ & $\begin{array}{l}0.202 \\
0.211\end{array}$ & $\begin{array}{l}0.237 \\
0.188\end{array}$ & $\begin{array}{l}0.121 \\
0.036\end{array}$ & $\begin{array}{l}0.275 \\
0.197\end{array}$ \\
\hline Plant height (cm) & $\begin{array}{l}r_{g} \\
r_{p}\end{array}$ & & & $\begin{array}{l}1 \\
1\end{array}$ & $\begin{array}{c}0.571 * * \\
0.296\end{array}$ & $\begin{array}{l}0.795 * * \\
0.646 * *\end{array}$ & $\begin{array}{l}0.705 * * \\
0.397 * *\end{array}$ & $\begin{array}{l}0.816 * * \\
0.693 * *\end{array}$ & $\begin{array}{l}0.999 * * \\
0.858 * *\end{array}$ & $\begin{array}{l}-0.165 \\
-0.046\end{array}$ & $\begin{array}{l}0.874 \\
0.744\end{array}$ \\
\hline $\begin{array}{l}\text { Effective tillers } \\
\text { per plant }\end{array}$ & $\begin{array}{l}r_{g} \\
r_{p}\end{array}$ & & & & $\begin{array}{l}1 \\
1\end{array}$ & $\begin{array}{c}0.418 * \\
0.086\end{array}$ & $\begin{array}{l}0.275 \\
0.091\end{array}$ & $\begin{array}{l}0.156 \\
0.113\end{array}$ & $\begin{array}{c}0.471 * * \\
0.247\end{array}$ & $\begin{array}{l}-0.419^{*} \\
-0.022\end{array}$ & $\begin{array}{l}0.251 \\
0.198\end{array}$ \\
\hline $\begin{array}{l}\text { Ear head length } \\
(\mathrm{cm})\end{array}$ & $\begin{array}{l}r_{g} \\
r_{p}\end{array}$ & & & & & $\begin{array}{l}1 \\
1\end{array}$ & $\begin{array}{l}0.971 * * \\
0.640 * *\end{array}$ & $\begin{array}{l}0.931 * * \\
0.790 * *\end{array}$ & $\begin{array}{l}0.819 * * \\
0.590 * *\end{array}$ & $\begin{array}{c}0.496 * * \\
0.326\end{array}$ & $\begin{array}{l}0.962 * * \\
0.738 * *\end{array}$ \\
\hline $\begin{array}{l}\text { Ear head girth } \\
(\mathrm{cm})\end{array}$ & $\begin{array}{l}r_{g} \\
r_{p}\end{array}$ & & & & & & $\begin{array}{l}1 \\
1\end{array}$ & $\begin{array}{l}0.931 * * \\
0.699 * *\end{array}$ & $\begin{array}{l}0.630 * * \\
0.465 * *\end{array}$ & $\begin{array}{c}0.711 * * \\
0.347\end{array}$ & $\begin{array}{l}0.858 * * \\
0.618 * *\end{array}$ \\
\hline $\begin{array}{l}1000 \text { grain weight } \\
\text { (g.) }\end{array}$ & $\begin{array}{l}r_{g} \\
r_{p}\end{array}$ & & & & & & & $\begin{array}{l}1 \\
1\end{array}$ & $\begin{array}{l}0.814 * * \\
0.728 * *\end{array}$ & $\begin{array}{l}0.579 * * \\
0.315\end{array}$ & $\begin{array}{l}0.998 * * \\
0.864 * *\end{array}$ \\
\hline $\begin{array}{l}\text { Biological } \\
\text { yield/plant (g) }\end{array}$ & $\begin{array}{l}r_{g} \\
r_{p}\end{array}$ & & & & & & & & $\begin{array}{l}1 \\
1\end{array}$ & $\begin{array}{l}-0.091 \\
-0.182\end{array}$ & $\begin{array}{l}0.908 * * \\
0.806 * *\end{array}$ \\
\hline $\begin{array}{l}\text { Harvest Index } \\
(\%)\end{array}$ & $\begin{array}{l}r_{g} \\
r_{p}\end{array}$ & & & & & & & & & $\begin{array}{l}1 \\
1\end{array}$ & $\begin{array}{c}0.336 \\
0.420 *\end{array}$ \\
\hline
\end{tabular}


Table.2 Direct (diagonal) and indirect (non-diagonal) effects of various characters on grain yield in maintainer lines of Pearl millet at genotypic $(\mathrm{G})$ and phenotypic $(\mathrm{P})$ levels

\begin{tabular}{|c|c|c|c|c|c|c|c|c|c|c|c|}
\hline Characters & & $\begin{array}{c}\text { Days to } \\
50 \% \\
\text { flowering }\end{array}$ & $\begin{array}{l}\text { Days to } \\
\text { maturity }\end{array}$ & $\begin{array}{c}\text { Plant } \\
\text { height } \\
(\mathrm{cm})\end{array}$ & $\begin{array}{c}\text { Effective } \\
\text { tillers per } \\
\text { plant }\end{array}$ & $\begin{array}{l}\text { Panicle } \\
\text { length } \\
\text { (cm) }\end{array}$ & $\begin{array}{l}\text { Panicle } \\
\text { girth } \\
(\mathrm{cm})\end{array}$ & $\begin{array}{l}\text { 1000- } \\
\text { grain } \\
\text { weight } \\
\text { (g) }\end{array}$ & $\begin{array}{l}\text { Biological } \\
\text { yield per } \\
\text { plant }(\mathrm{g})\end{array}$ & $\begin{array}{c}\text { Harvest } \\
\text { Index } \\
(\%)\end{array}$ & $\begin{array}{c}\text { Correlation } \\
\text { with grain } \\
\text { yield per } \\
\text { plant (g) }\end{array}$ \\
\hline $\begin{array}{l}\text { Days to } 50 \% \\
\text { flowering }\end{array}$ & $\begin{array}{l}\mathrm{G} \\
\mathrm{P}\end{array}$ & $\begin{array}{c}0.49014 \\
-0.00874\end{array}$ & $\begin{array}{c}-0.44670 \\
0.01246\end{array}$ & $\begin{array}{l}-0.18465 \\
-0.01412\end{array}$ & $\begin{array}{c}0.00796 \\
-0.00040\end{array}$ & $\begin{array}{l}0.02009 \\
0.00140\end{array}$ & $\begin{array}{l}-0.01668 \\
-0.00232\end{array}$ & $\begin{array}{c}-0.00426 \\
0.01429\end{array}$ & $\begin{array}{l}0.27307 \\
0.13720\end{array}$ & $\begin{array}{l}0.08451 \\
0.02821\end{array}$ & \\
\hline Days to maturity & $\begin{array}{l}\mathrm{G} \\
\mathrm{P}\end{array}$ & $\begin{array}{c}0.48692 \\
-0.00814\end{array}$ & $\begin{array}{c}-0.44966 \\
0.01337\end{array}$ & & & & $\begin{array}{l}-0.00972 \\
-0.00104\end{array}$ & & & & \\
\hline $\begin{array}{l}\text { Plant height } \\
\text { (cm) }\end{array}$ & $\begin{array}{l}\mathrm{G} \\
\mathrm{P}\end{array}$ & $\begin{array}{c}0.14855 \\
-0.00230\end{array}$ & $\begin{array}{c}-0.16519 \\
0.00382\end{array}$ & $\begin{array}{l}-0.60927 \\
-0.05359\end{array}$ & $\begin{array}{r}0.03867 \\
-0.00127\end{array}$ & $\begin{array}{l}0.07444 \\
0.01207\end{array}$ & $\begin{array}{l}-0.13534 \\
-0.01780\end{array}$ & $\begin{array}{c}-0.02015 \\
0.05203\end{array}$ & $\begin{array}{l}1.62179 \\
0.77748\end{array}$ & $\begin{array}{l}-0.07982 \\
-0.02622\end{array}$ & $\begin{array}{l}0.874 \\
0.744\end{array}$ \\
\hline $\begin{array}{l}\text { Effective tillers } \\
\text { per plant }\end{array}$ & $\begin{array}{l}\mathrm{G} \\
\mathrm{P}\end{array}$ & $\begin{array}{c}0.05762 \\
-0.00081\end{array}$ & & & & & & & & & \\
\hline $\begin{array}{l}\text { Panicle length } \\
\text { (cm) }\end{array}$ & $\begin{array}{l}\mathrm{G} \\
\mathrm{P}\end{array}$ & $\begin{array}{c}0.10512 \\
-0.00066\end{array}$ & & & & & $\begin{array}{l}-0.20563 \\
-0.02865\end{array}$ & $\begin{array}{c}-0.02544 \\
0.05936\end{array}$ & $\begin{array}{l}1.32625 \\
0.53727\end{array}$ & $\begin{array}{l}0.23997 \\
0.18561\end{array}$ & $\begin{array}{l}0.962 * * \\
0.738 * *\end{array}$ \\
\hline $\begin{array}{l}\text { Panicle girth } \\
\text { (cm) }\end{array}$ & $\begin{array}{l}\mathrm{G} \\
\mathrm{P}\end{array}$ & $\begin{array}{r}0.04260 \\
-0.00045\end{array}$ & $\begin{array}{c}-0.02276 \\
0.00031\end{array}$ & $\begin{array}{l}-0.42966 \\
-0.02129\end{array}$ & $\begin{array}{c}0.01858 \\
-0.00039\end{array}$ & $\begin{array}{l}0.10037 \\
0.01195\end{array}$ & $\begin{array}{l}-0.19192 \\
-0.04480\end{array}$ & $\begin{array}{c}-0.02298 \\
0.05248\end{array}$ & $\begin{array}{l}1.01933 \\
0.42303\end{array}$ & $\begin{array}{l}0.34409 \\
0.19765\end{array}$ & $\begin{array}{l}0.858 * * \\
0.618 * *\end{array}$ \\
\hline $\begin{array}{l}\text { 1000-grain } \\
\text { weight (g) }\end{array}$ & $\begin{array}{l}\mathrm{G} \\
\mathrm{P}\end{array}$ & $\begin{array}{c}0.08468 \\
-0.00166\end{array}$ & $\begin{array}{c}-0.09104 \\
0.00282\end{array}$ & $\begin{array}{l}-0.49729 \\
-0.03713\end{array}$ & $\begin{array}{c}0.01055 \\
-0.00048\end{array}$ & $\begin{array}{l}0.09656 \\
0.01477\end{array}$ & $\begin{array}{l}-0.17867 \\
-0.03130\end{array}$ & $\begin{array}{c}-\mathbf{0 . 0 2 4 6 8} \\
0.07510\end{array}$ & $\begin{array}{l}1.31810 \\
0.66242\end{array}$ & $\begin{array}{l}0.28011 \\
0.17900\end{array}$ & $\begin{array}{l}0.998 * * \\
0.864 * *\end{array}$ \\
\hline $\begin{array}{l}\text { Biological yield } \\
\text { per plant (g) }\end{array}$ & $\begin{array}{l}\mathrm{G} \\
\mathrm{P}\end{array}$ & $\begin{array}{c}0.08268 \\
-0.00132\end{array}$ & $\begin{array}{c}-0.10653 \\
0.00251\end{array}$ & $\begin{array}{l}-0.61037 \\
-0.04578\end{array}$ & $\begin{array}{c}0.03187 \\
-0.00106\end{array}$ & $\begin{array}{l}0.07674 \\
0.01103\end{array}$ & $\begin{array}{l}-0.12084 \\
-0.02082\end{array}$ & $\begin{array}{c}-0.02010 \\
0.05465\end{array}$ & $\begin{array}{l}1.61889 \\
0.91026\end{array}$ & $\begin{array}{l}-0.04406 \\
-0.10340\end{array}$ & $\begin{array}{l}0.908 * * \\
0.806 * *\end{array}$ \\
\hline $\begin{array}{l}\text { Harvest Index } \\
(\%)\end{array}$ & $\begin{array}{l}\mathrm{G} \\
\mathrm{P}\end{array}$ & $\begin{array}{c}0.08561 \\
-0.00043\end{array}$ & $\begin{array}{c}-0.05422 \\
0.00048\end{array}$ & $\begin{array}{l}0.10052 \\
0.00247\end{array}$ & $\begin{array}{c}-0.02832 \\
0.00009\end{array}$ & $\begin{array}{l}0.04646 \\
0.00610\end{array}$ & $\begin{array}{c}0.13650 \\
-0.01557\end{array}$ & $\begin{array}{c}-0.01429 \\
0.02364\end{array}$ & $\begin{array}{l}-0.14743 \\
-0.16551\end{array}$ & $\begin{array}{l}0.48380 \\
0.56869\end{array}$ & $\begin{array}{c}0.336 \\
0.420^{*}\end{array}$ \\
\hline
\end{tabular}


The genotypic and phenotypic correlation coefficients of grain yield with its contributing traits in maintainer lines of pearl millet were partitioned into direct and indirect effects through path coefficient analysis is depicted in Table 2. The correlation coefficient indicates the absolute totally correlated with various characters whereas path coefficient revealed the related to importance of various component characters on grain yield. In the present investigation, path coefficient analysis was carried out at genotypic as well as phenotypic levels by taking grain yield per plant as a dependent variable and rest of the characters are independent variables.

At genotypic level, highest positive direct effect on grain yield per plant was recorded for biological yield per plant (1.61889) followed by days to $50 \%$ flowering (0.49014), harvest index (0.48380), ear head length $(0.09368)$ and effective tillers per plant (0.06769); whereas, highest negative direct effect was recorded for plant height ($0.60927)$, days to maturity (-0.44996), ear head girth $(-0.19192)$ and 1000 grain weight $(-0.02468)$, in maintainer lines of pearl millet.

At phenotypic level, highest positive direct effect on grain yield per plant was observed for biological yield per plant $(0.91026)$ followed by harvest index (0.56869), 1000 grain weight (0.07510), ear head length (0.01869) and days to maturity (0.01337); whereas, highest negative direct effect was observed for plant height (-0.05359), ear head girth (-0.04480), days to $50 \%$ flowering ($0.00874)$ and effective tillers per plant (0.00428), in maintainer lines of pearl millet.

The path coefficient based on genotypic and phenotypic correlation coefficient revealed that the highest positive correlation of grain yield per plant was observed with 1000-grain weight, ear head length, biological yield per plant, plant height, ear head girth and harvest index. The highest direct effect was recorded in biological yield per plant followed by harvest index, days to $50 \%$ flowering and ear head length; it reflects a true relationship between them and selection can be practiced for these characters in order to improve grain yield in pearl millet. The positive association of grain yield per plant with days to maturity, plant height, effective tillers per plant, ear head girth and 1000-grain weight is mainly due to indirect effect of these traits through another component characters; therefore, selection for latter characters through which the indirect effect is exerted may be effective.

Thus, biological yield per plant, harvest index, days to $50 \%$ flowering and ear head girth in this study has been identified as major selection criteria for obtaining good parental line. Hence, main emphasis should be given to these traits in pearl millet breeding programme. Same results were reported by Chaudhary et al., (2003), Arulselvi et al., (2008), Kumar et al., (2014), and Bhasker et al., (2017) also reported that at genotypic level the fodder yield per plot had exhibited largest direct effect on grain yield per plant followed by panicle length, 1000-grain weight and number of productive tillers per plant.

\section{Acknowledgement}

I would like to thank Department of Plant Breeding and Genetics, S.K.N College of Agriculture, Jobner (Jaipur) Rajasthan for providing all the materials and facilities throughout the course of this research work. I would also like to express my sincere gratitude to Dr. S.S. Rajput, Mr. Giradhari Lal Yadav for their whole hearted support and guidance throughout the research work.

\section{References}

Abuali, A.I., Awadalla, A.A. and Atif, E.I. 2012. Character association and path analysis in pearl millet (Pennisetum 
glaucum L.). American Journal of Experimental Agriculture, 2(3): 370-381.

Anonymous, 2019. Project Coordinator Review. Pearl Millet News (March, 2019), ICARAICRP on Pearl Millet, Jodhpur, 8: 1.

Arulselvi, S., Mohanasundaram, K. and Selvi, B. 2008. Correlation and path analysis for grain yield and its components in pearl millet (Pennisetum glaucum (L.) R. Br.). Madras Agricultural Journal, 95 (7-12): 311-314.

Bhasker, K., Shashibhushan, D., Murali, K.K. and Bhave, M.H.V. 2017. Correlation and path analysis for grain yield and its components in Pearl Millet [Pennisetum glaucum (L). R. Br.]. Bulletin of Environment, Pharmacology and Life Sciences, 6(1): 104-106.

Chaudhary, M.H., Subhani, G.M., Shaheen, M.S. and Saleem, U. 2003. Correlation and path coefficients analysis in pearl millet (Pennisetum glaucum L.). Pakistan Journal of Biological Sciences, 6(6): 597600.

Dewey, D.I. and Lu, K.H. 1959. A correlation and path coefficient analysis of components of crested wheat grass seed production. Agronomy Journal, 51: 515518.

Fleck, H. 1981. Introduction to nutrition. Macmillan Publishing Co., India, 49.

Govidaraj, M., Selvi, B. and Rajarathinam, S. 2009. Correlation studies for grain yield components and nutritional quality traits in pearl millet [Pennisetum glaucum (L.) R. Br.] germplasm. World Journal of Agricultural Sciences, 5(6): 686-689.

Gupta, S.K., Nepolean, T, Sankar, S.M, Rathore, A, Das, R.R, Rai, K.N. and Hash, C.T. 2015. Patterns of molecular diversity in current and previously developed hybrid parents of pearl millet [Pennisetum glaucum (L.) R. Br.]. American Journal Plant Science, 6: 16971712.

Johnson, H.W., Robinson, H.F. and Comstock, R.E. 1955. Estimation of genetic and environmental variability in soybeans. Agronomy Journal, 47: 314-318.

Kumar, R., Harish, S., Dalal, M.S., Malik, V., Devvart., Chugh, L.K., Garg, P. and Raj, K. 2014. Studies on variability, correlation and path analysis in pearl millet genotypes. Forage Research, 40(3): 163-167.

Pandy, S. and E.T., Gritton 1975. Genotypic and phenotypic variance and correlation in peas. Crop Science, 15: 353-356.

Patil, S.K., Gupta, K., Dangi, K.S., Shashibhushan, D., Balram, M. and Ramesh, T. 2018. Panicle traits and plant height are important Selection Indices to enhance productivity in pearl millet [Pennisetum glaucum (L.) R. Br.] populations. International Journal of Current Microbiology and Applied Sciences, 7(12): 306-312.

Rasitha, R., Iyanar, K., Ravikesavan, R. and Senthil, N. 2019. Studies on genetic parameters, correlation and path analysis for yield attributes in the maintainer and restorer lines of pearl millet [Pennisetum glacum (L.) R. Br.]. Electronic Journal of Plant Breeding, 10(2): 382-388.

Singh, R.K. and Chaudhary, B.D. 1985. Biometrical Methods in Quantitative Genetic Analysis. Third edition. Kalyani publisher, New Delhi.

Vavilov, N.I. 1950. The origin, variation, immunity and breeding of cultivated plants. Chronica Botanika, 13: 1-366.

\section{How to cite this article:}

Sundar Lal Dadarwal, Shyam Singh Rajput and Giradhari Lal Yadav. 2020. Studies on Correlation and Path Analysis for Grain Yield and its Components in Maintainer Lines of Pearl Millet [Pennisetum glaucum (L.) R. Br.]. Int.J.Curr.Microbiol.App.Sci. 9(12): 1158-1164. doi: https://doi.org/10.20546/ijcmas.2020.912.140 